\title{
Contribution de la Régénération Naturelle Assistée des ligneux dans l'approvisionnement en bois des ménages dans le département de Magaria (Niger)
}

\author{
Mahamane LARWANOU ${ }^{1 *}$, Adamou Mahamane MOUSTAPHA ${ }^{1}$, \\ Mahaman Lawali $\mathrm{RABE}^{2}$ et Danguimbo IRO ${ }^{2}$ \\ ${ }^{1}$ African Forest Forum (AFF), clo World Agroforestry Centre (ICRAF), United Nations Avenue, Gigiri, P.O. \\ Box 30677 - 00100 Nairobi, Kenya. Tel: +254207224624; Fax: +254207224001. \\ ${ }^{2}$ Faculté d'Agronomie, Université Abdou Moumouni de Niamey, BP 10960, Niamey, Niger. \\ *Auteur correspondant, E-mail: m.larwanou@cgiar.org
}

\section{RESUME}

Cet article présente les impacts de la Régénération Naturelle Assistée (RNA) sur l'approvisionnement en bois des ménages dans deux terroirs villageois (Arasofoua et Gaounawa) du département de Magaria au Niger. L'objectif visé était d'évaluer la quantité de bois utilisée au niveau des ménages pour les différents usages (bois énergie, construction, et service). La méthodologie utilisée pour atteindre cet objectif a consisté en des enquêtes auprès des ménages sur le type de bois utilisé et leur quantité ainsi que des mesures pour évaluer le changement induit à travers la pratique de la Régénération Naturelle Assistée. Les résultats obtenus montrent que les populations de ces villages arrivent à satisfaire leurs besoins en bois de feu et de service. Cette technique est pratiquée depuis 1994 dans ces villages. Chaque exploitant collecte en moyenne 24,9 et $17,5 \mathrm{~m}^{3}$ de bois par an dans les deux terroirs villageois respectivement en fonction des différents types d'usage (chauffe, construction, ustensiles de cuisine, etc.) et types de bois utilisés. Environ 90,8\% des exploitants n'achètent pas le bois. L'autoconsommation moyenne en bois dans les deux villages est de 57,8\%. Les transhumants et les coupes frauduleuses, constituent les contraintes majeures de cette pratique. Sensibiliser et former davantage les paysans aux techniques de régénération et impliquer les femmes dans cette nouvelle pratique favoriseraient la constitution de parcs agroforestiers.

(c) 2012 International Formulae Group. All rights reserved.

Mots clés : Innovation, ménages, produits ligneux, Sahel, Niger.

\section{INTRODUCTION}

$\mathrm{Au}$ cours des années 70 et 80 , de nombreuses publications ont été consacrées à la crise d'énergie dans les pays du Sahel et ailleurs dans les zones arides et semi-arides (Eckholm, 1975 ; Winterbotton, 1980). Cette crise résultait d'un fort déficit entre les besoins en énergie de la population, qui étaient presque exclusivement fournis par le bois, et la faible productivité des formations ligneuses. En même temps, le Sahel subissait deux périodes de sécheresse successives. Ces auteurs en déduisaient que dans un avenir proche, des zones entières à la périphérie des 
villes au Sahel seraient complètement déboisées à cause des besoins de bois de feu de leurs populations dont le nombre augmentait rapidement.

Cette dégradation s'est traduite par une réduction, voire une disparition du couvert arboré et arbustif dans de nombreux villages (Larwanou, 1998 ; Joet et al., 1998 ; Carucci, 2000). Cette régression de la ressource ligneuse a de multiples répercussions, notamment la baisse de la fertilité des sols, l'accentuation de l'érosion hydrique et éolienne, la difficulté d'approvisionnement en bois de feu et de service (Jouve, 1991; Awaiss, 2000 ).

Ainsi, plus le bois de feu devenait rare, plus les femmes parcouraient de longues distances à sa recherche, ou étaient obligées d'utiliser les résidus des cultures et les bouses de vache desséchées. Le bois de construction devenait aussi rare, provoquant une augmentation insupportable pour le budget du paysan sahélien.

Pour faire face à cette situation au Sahel en général et au Niger en particulier, diverses stratégies collectives et individuelles de protection de l'environnement ont été entreprises avec l'appui de l'assistance internationale et bilatérale (Jesse, 1995; Larwanou et al, 2006; Abdourahmane, 2009). Ainsi, dans certains pays sahéliens (Sénégal, Mali, Burkina Faso, Niger, Tchad), il a été dénombré entre vingt et trente projets dont l'objectif principal était la gestion des terroirs et l'atténuation de la dégradation des ressources naturelles (Jouve, 1996). Des solutions ont été développées pour faire face aux problèmes de dégradation des terres et pour améliorer les systèmes de production (Yamba, 1994): aménagement forestier, Régénération Naturelle Assistée (RNA), multiplication végétative à faible coût (MVfc) (Peltier et al., 1994 ; Bertrand et al., 1999 ; Bellefontaine, 2005; Bationo et al., 2005).
La RNA et la MVfc se basent sur divers rejets naturels ou artificiels et semis qui poussent naturellement dans les champs. Ces rejets sont sélectionnés, partiellement élagués et protégés. Beaucoup de projets et services techniques de l'Etat ont vulgarisé cette innovation au Niger, notamment dans le département de Magaria. Le défrichement amélioré, qui est une variante de la Régénération Naturelle Assistée (Banoin et al., 1994, Bonkano, 2005 ), est couramment pratiqué. La Régénération Naturelle Assistée a été développée et adoptée par les populations locales (Marou et al., 2002).

C'est pour suivre les impacts de la pratique de cette technique dans la vie des ménages que cette étude a été conduite en 2008 dans deux terroirs villageois du département de Magaria avec comme objectif principal d'évaluer les utilisations et la quantité de bois au niveau des ménages provenant des champs des producteurs à travers la Régénération Naturelle Assistée.

\section{MATERIEL ET METHODES Sites d'étude}

Les terroirs de Gaounawa et Arasofoua se situent dans le chef-lieu du département de Magaria (Figure 1), à environ $95 \mathrm{~km}$ de la ville de Zinder. La densité de la population est de 57,6 habitants au $\mathrm{km}^{2}$, une des plus élevées du Niger. Les terroirs de ces deux villages ont une population estimée respectivement à 1375 et 1198 habitants. Elle est composée de plusieurs ethnies, dont les Haoussa, majoritaires. Le village d'Arasofoua est situé à une altitude moyenne de $405 \mathrm{~m}$ et celui de Gaounawa à $413 \mathrm{~m}$.

Le climat est de type sahélien avec une saison sèche qui se subdivise en saison froide (novembre à février), en saison sèche chaude (mars à mai) et une saison pluvieuse de juin à octobre. L'agriculture représente la principale activité (à plus de 95\%) des populations. Elle est pratiquée sous deux régimes, le pluvial 
M. LARWANOU et al. / Int. J. Biol. Chem. Sci. 6(1): 24-36, 2012

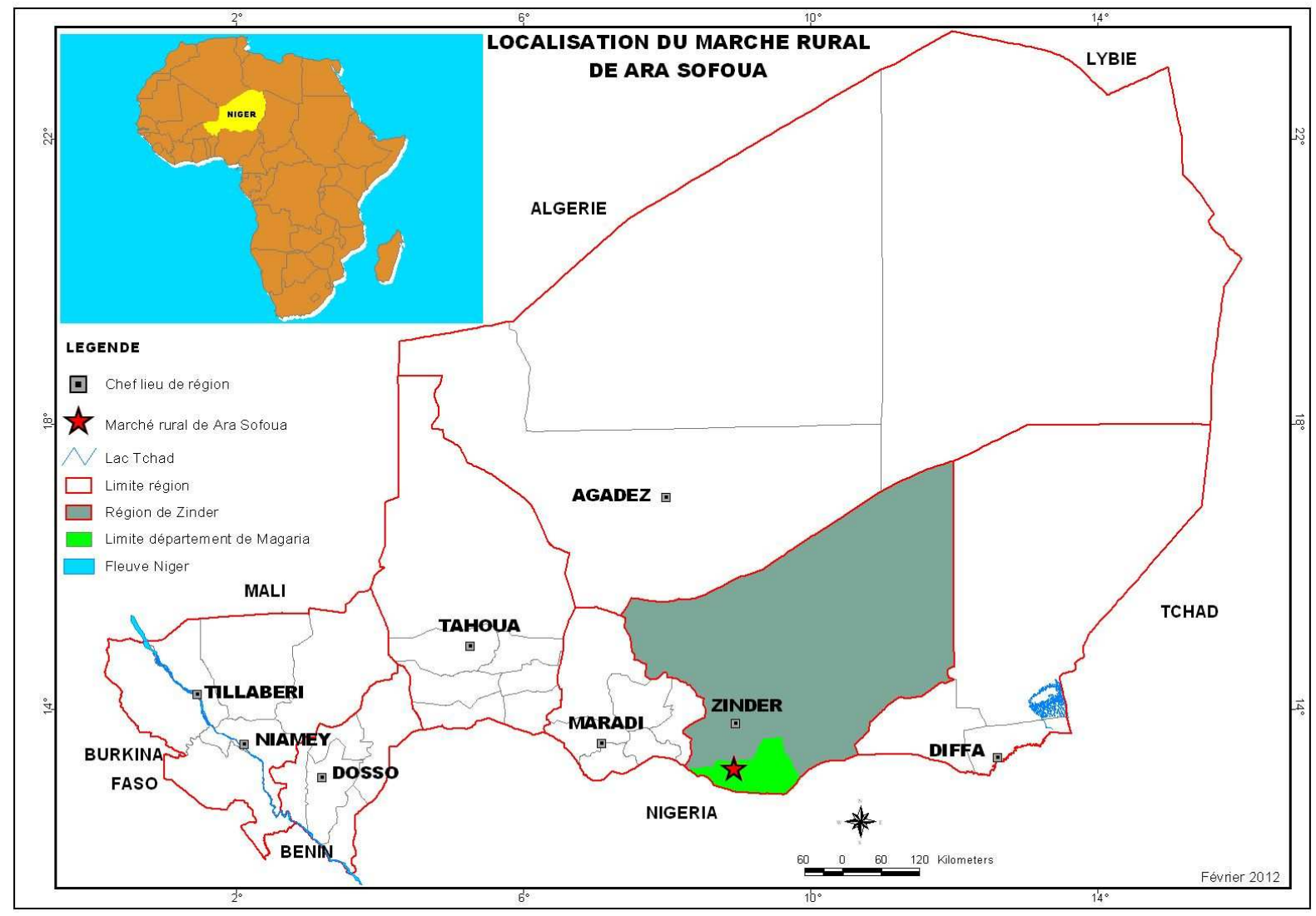

Figure 1 : Carte du Niger avec le département de Magaria. Source : Diagnostic villageois (2005), OSE/IL/ED. 
durant la saison des pluies de juin à octobre et le régime irrigué durant la saison sèche de novembre à mai.

\section{Collecte et analyse des données}

Pour la typologie de la végétation, des observations ont été faites dans les deux terroirs villageois couplées aux interviews auprès de la population.

Les données ont été collectées de juillet à septembre 2008 à l'aide de deux fiches dont la première a servi à réunir les informations sur la pratique de la RNA et la seconde pour la quantification et les usages du bois dans les ménages.

Les assemblées villageoises organisées dans les deux villages ont permis de recenser respectivement 137 et 157 ménages à Arasofoua et à Gaounawa parmi lesquels 10\% des chefs de ménage (14 et 16) ont été choisis aléatoirement pour l'étude. Ces chefs de ménage sont tous des hommes, car la coutume locale n'accepte pas de responsabiliser une femme comme chef de ménage. Les chefs de ménage constituant l'échantillon ont été interviewés pour la collecte des informations. Ces dernières avaient trait à la détermination de la provenance du bois utilisé dans et autour des maisons, à la distance parcourue pour sa recherche, aux moyens de transport, aux types d'objets fabriqués ou utilisés, aux usages.

Les différents objets recensés ont fait l'objet de mesures pour déterminer leur proportion au sein des ménages et à l'échelle du village, leur volume, ainsi que les espèces utilisées. La détermination du volume de bois utilisé se basait sur la formule de Huber $\mathrm{V}=1 / 4(3,14) * \mathrm{~d}^{2} \mathrm{~m} * 1$,

avec $\mathrm{dm}$ : diamètre médian (en mètre); $\mathrm{V}$ : volume médian (en mètre-cube) ; 1 : longueur totale (en mètre).

Le nombre total d'objets pour chaque type d'usage au niveau des ménages a été déterminé.

Pour chaque utilisation (bois de feu, piquet, etc.), les mesures ont pris en compte environ $12 \%$ de leur nombre total. Une extrapolation de l'échantillon au nombre total a permis d'estimer le volume total.

La quantité en stère (unité de volume occupée par des bûches, empilées dans un mètre cube) est utilisée pour la conversion du volume en $\mathrm{m}^{3}$. C'est la seule valeur légale commerciale.

Les données collectées ont été analysées avec le logiciel SPSS (Statistical Package for Social Sciences) pour les analyses descriptives.

\section{RESULTATS}

Evolution et typologie de la végétation

La végétation dans la zone d'étude était caractérisée dans les années 1970 et antérieures (Saadou, 1990) par la présence de formations naturelles (forêts classées) et d'espaces communautaires ou aires de pâturage.

Cette structuration de la végétation a évolué au fil des années par une transformation progressive dans certains cas, et brusque dans d'autres, pour laisser place aux parcs agroforestiers (arbres dispersés dans les champs). L'augmentation de la population et le besoin pressant de terres de cultures sont à la base de cette évolution récente.

Le couvert végétal est passé d'une structure plus ou moins fermée (dans les années 1950) à une structure plus ouverte, caractérisée par des cimes ne se touchant qu'occasionnellement. Cette structuration est façonnée pour permettre une meilleure pénétration de la lumière pour les cultures annuelles. La disparition totale de beaucoup d'espèces ligneuses est à déplorer. Larwanou (1998) en a recensé 26 dans le département de Magaria lors d'une première enquête sur les espèces ligneuses disparues et/ou menacées de disparition.

Aujourd'hui, on rencontre des espèces très caractéristiques telles que Prosopis africana, Faidherbia albida, Piliostigma reticulatum et Parkia biglobosa suivant les stations écologiques qui sont caractéristiques ou typiques des différents parcs agroforestiers présents dans la zone. Ceux-ci ont des 
caractéristiques monospécifiques (avec une seule espèce dominante) ou plurispécifiques (un mélange de plusieurs espèces en proportions presque égales). Ainsi, d'une manière générale, dans le département de Magaria, cinq types de parcs peuvent être retenus:

- parcs à $F$. albida épars ;

- parcs à $F$. albida dense

- parcs à F.albida et $P$. africana ;

- parcs à $F$. albida, $P$. africana et $P$. reticulatum ;

- parcs à F.albida et $P$. reticulatum.

Il est important de souligner que ces parcs agroforestiers ont été construits par la population. Certaines espèces ont été sélectionnées, préservées, façonnées et gérées (Larwanou et al., 2006). Chaque pied de F.albida et de P.reticulatum est élagué. Ces espèces sont protégées et épargnées de toute forme d'exploitation anarchique parce qu'elles présentent certaines qualités aux yeux de la population. Non seulement, elles contribuent à l'amélioration de la fertilité des sols et augmentent ainsi les rendements agricoles mais elles fournissent du fourrage pour le bétail ou entrent dans l'alimentation humaine et surtout procurent des revenus pour les paysans à travers la vente du bois, des fruits, des feuilles. Certaines parties de ces espèces sont utilisées dans la pharmacopée traditionnelle. Malgré l'exploitation du potentiel ligneux pour différents usages, force est de constater que la densité des arbres a évolué positivement dans les terroirs (Figure 2). Dans certains terroirs, il a été dénombré plus de 50 pieds à l'hectare dans les champs dans cette partie du Niger grâce à l'entretien et de la protection de la régénération naturelle (Larwanou et al., 2006).

Distance parcourue pour
l'approvisionnement en bois
Le Tableau 1 suivant indique les
distances parcourues pour le ramassage de
bois mort ou frais selon les producteurs. La
collecte de bois est généralement assurée par
les femmes et les enfants pour

l'autoconsommation. Les hommes interviennent en cas de grands travaux (construction, grenier, clôture, cases, hangars, etc.). La distance moyenne est de 1,75 $\pm 0,46$ $\mathrm{km}$ à Arasofoua et 3,02 $\pm 0,8 \mathrm{~km}$ à Gaounawa (Tableau 1). D'après les paysans interrogés, ces distances sont relativement moindres comparées aux années 1980.

Le bois provient des défriches, élagage des arbres, coupe de branches mortes et/ou vivantes. Il est à souligner que tout le bois collecté provient des champs et parfois des jardins, car il n'existe plus de forêts.

\section{Utilisation du bois en fonction de leurs dimensions}

Le type de bois utilisé dans chaque ménage dépend des types d'usages envisagés. Dans notre étude, la longueur et le diamètre des différents objets observés dans les ménages, selon les types d'usages, sont les variables qui ont été prises en compte. Le Tableau 2 ci-dessous montre les différentes utilisations des bois en fonction de leurs dimensions.

Selon son emplacement sur l'arbre et l'âge, le bois utilisé a des tailles variables qui déterminent le type d'utilisation. Certains types de bois comme celui de chauffe sont coupés et transportés sans délais près des habitations, stockés pour des utilisations futures par crainte de se faire voler dans les champs. Le bois ainsi conservé par le chef d'exploitation est utilisé pour la cuisson et la construction (clôture, grenier, etc.). La nature (espèce, diamètre, longueur) du bois varie d'un usage à l'autre.

Les objets utilisés dans les deux villages varient selon les besoins préférentiels de chaque ménage. Le Tableau 3 ci-dessous indique la proportion des différents types d'usages classés par ordre d'importance dans les deux villages.

L'utilisation du bois pour les clôtures $(69,52 \%)$ et les constructions $(67,24 \%)$ est la plus élevée au niveau des villages. Elle est en phase avec le mode de vie en milieu rural 
sahélien où toutes les habitations sont érigées à base du bois.

En effet, dans les terroirs villageois où il n'y a pas d'argile pour la confection des clôtures, la proportion de bois de service utilisée est très élevée, notamment pour le renouvellement des toitures tous les six ans. Le bois de $P$. africana, très résistant, peut durer longtemps dans le sol sans subir de modifications telles que pourriture et attaques des termites.

La quantité du bois utilisée dépend de son usage. Le Tableau 4 indique les volumes totaux de bois par usage et par ménage dans les deux villages. Les objets comme l'échelle, le lit, le mortier, le pilon, le support de calebasse, la chaise et les piquets à linge sont les moins utilisés.

Le volume total de bois utilisé au sein d'un village augmente en fonction de la quantité de bois nécessaire pour les clôtures de chaque concession. Ainsi, le nombre de piquets utilisés varie d'au moins 12 piquets pour des ménages ayant une clôture en banco à plus de 111 piquets par ménage pour la clôture en paille.

Le volume de bois au niveau du village de Arasofoua $\left(\mathrm{V}=3481 \mathrm{~m}^{3}\right)$ est plus élevé que celui de Gaounawa $\left(\mathrm{V}=2763 \mathrm{~m}^{3}\right)$. Cette nette différence (environ $718 \mathrm{~m}^{3}$ ) est certainement due à la présence d'un marché de bois à Gaounawa qui leur permet d'écouler facilement leurs produits ligneux. Si l'on compare ces volumes au nombre d'habitants par village, on obtient respectivement $2,01 \mathrm{~m}^{3}$ pour Gaounawa et $2,91 \mathrm{~m}^{3}$ pour Arasofoua qui compte 177 habitants de moins. La réserve en bois est apportée et entassée autour des maisons par crainte des voleurs. Les espèces les plus exploitées dans ces terroirs villageois sont $P$. africana, A. indica, $H$. indica et $F$. indica (Tableau 5). D'après l'enquête, les espèces à forte régénération sont les plus utilisées.

Le bois de chaque espèce est coupé en fonction de sa dernière finition (usage). Les paysans préfèrent conserver sur pied les espèces les plus utiles et qui ne compétaient pas avec les cultures pour les ressources.

La consommation moyenne en bois de feu est de l'ordre de 3 stères par mois et par ménage au niveau des deux villages durant la période de l'enquête. Cette consommation diffère suivant les ménages et les villages. Il est important de préciser que ce volume est un volume minimal, car l'étude a été conduite lors des travaux champêtres, période non propice à la collecte de bois. Les besoins d'énergie domestique varient en fonction de la période de l'année, de la taille des ménages et des habitudes culinaires (Eckholm, 1975). L'autoconsommation moyenne en bois a été évaluée à environ $58 \%$ pour les deux terroirs villageois, le solde est vendu sur les marchés locaux.

L'estimation du bois de service (toiture des maisons, case, clôture, grenier) révèle un volume moyen variable dans chaque classe d'usage (Tableau 4). Ainsi :

- pour la construction des maisons, trois types de bois sont utilisés : la grande charpente, les traverses, les ossatures;

- $\quad$ pour la clôture, il est utilisé au moins 85 piquets en moyenne pour chaque propriété ; l'espèce $P$. africana est la plus utilisée grâce à sa résistance aux termites et à la pourriture. Cet usage contribue à une forte consommation de cette espèce ;

- $\quad$ pour les greniers, trois à quatre types de bois sont utilisés : la petite charpente en bois de $P$. africana ou d'Hyphaene thebaica, les traverses et les ossatures. Les tours des greniers sont faits à base du bois de G. senegalensis grâce à son élasticité et aussi $P$. reticulatum, Combretum micrantum ou encore Azadirachta indica ;

- pour la construction des cases, ce sont les branches vertes aisées à travailler de $P$. reticulatum, $G$. senegalensis, etc., qui sont les plus utilisées. Ces branches ont en moyenne une longueur de $1,30 \mathrm{~m}$ et un diamètre d'environ $8 \mathrm{~cm}$. Il est à noter que le support des cases est dans la plupart des cas construit en banco réduisant ainsi l'utilisation du bois. La commercialisation 
du bois s'observe principalement à Gaounawa à cause du marché de bois. Le surplus du bois exploité à Arasofoua est écoulé dans ce marché et celui de Bandé.

Les ustensiles de cuisine et les outils aratoires sont façonnés à base du bois des espèces ligneuses comme $P$. africana,

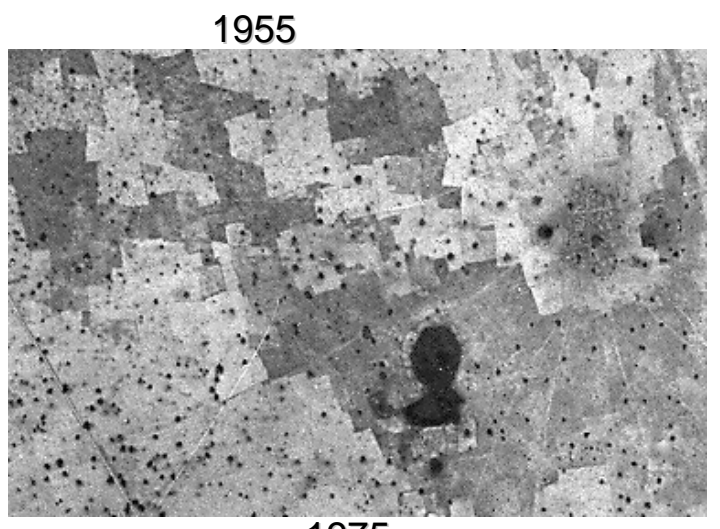

1975
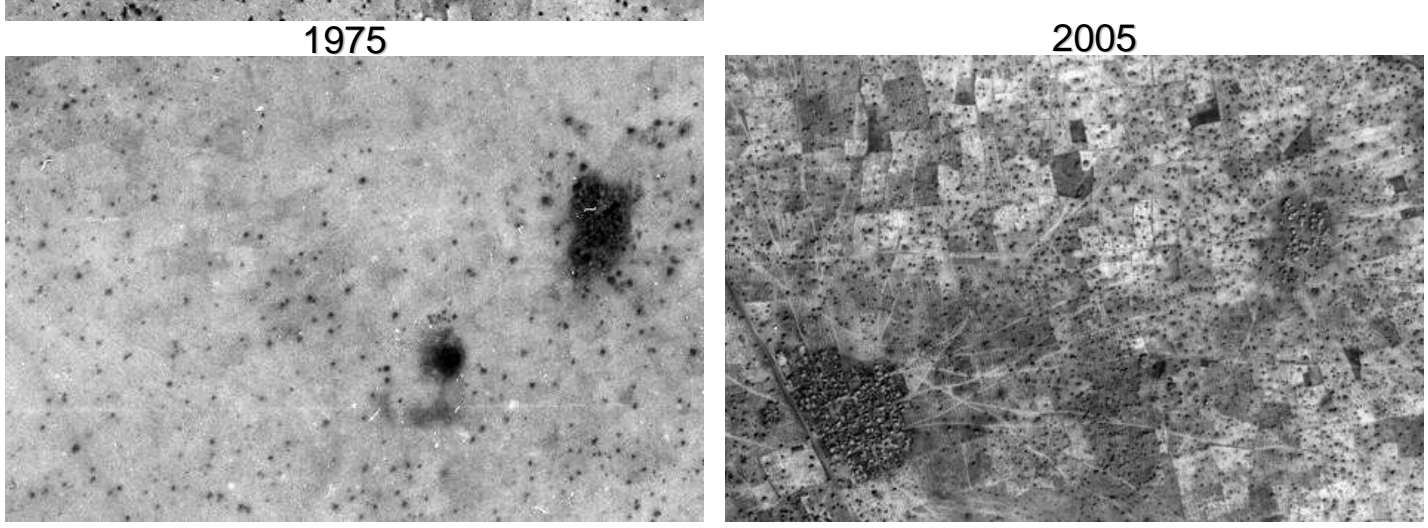

Figure 2: Un terroir villageois dans le département de Magaria au Niger en 1955, 1975 et 2005. Les petits points noirs sont les arbres qui augmentent en nombre avec l'accroissement de la population du village.

Tableau 1 : Distance $(\mathrm{km})$ parcourue et la proportion (\%) des producteurs pour la collecte du bois au niveau des deux villages.

\begin{tabular}{|c|c|c|c|c|c|}
\hline & \multicolumn{4}{|c|}{ Classes (km) } & Moyenne (km) \\
\hline Arasofoa & {$[1-1,75[$} & {$[1,75-2,50[$} & {$[2,50-3,25[$} & $>3,25$ & $1,75 \pm 0,46$ \\
\hline $\begin{array}{l}\text { Proportion (\%) des } \\
\text { producteurs }\end{array}$ & 53,4 & 27,5 & 18,9 & 0,2 & \\
\hline Gaounawa & [2-3[ & {$[3-4[$} & {$[4-5[$} & $>5$ & $3,02 \pm 0,80$ \\
\hline $\begin{array}{l}\text { Proportion (\%) des } \\
\text { producteurs }\end{array}$ & 38,6 & 46,5 & 9,6 & 5,3 & \\
\hline
\end{tabular}


Tableau 2 : Type d'objets utilisés et les dimensions dans les ménages au niveau des deux villages.

\begin{tabular}{lcccc}
\hline Type d'objets & \multicolumn{2}{c}{ Arasofoa } & \multicolumn{2}{c}{ Gaounawa } \\
\cline { 2 - 5 } & $\begin{array}{c}\text { Diamètre } \\
\text { moyen }(\mathbf{c m})\end{array}$ & $\begin{array}{c}\text { Longueur moyenne } \\
(\mathbf{m})\end{array}$ & $\begin{array}{c}\text { Diamètre moyen } \\
(\mathbf{c m})\end{array}$ & $\begin{array}{c}\text { Longueur } \\
\text { moyenne }(\mathbf{m})\end{array}$ \\
\hline Billes & $23,21 \pm 0,08$ & $1,03 \pm 0,25$ & $21,31 \pm 0,09$ & $0,81 \pm 0,44$ \\
Billons & $10,02 \pm 0,02$ & $0,80 \pm 0,32$ & $9,43 \pm 0,01$ & $0,82 \pm 0,55$ \\
Bois fins & $2,17 \pm 0,001$ & $1,32 \pm 0,24$ & $2,77 \pm 0,03$ & $1,26 \pm 0,27$ \\
Branches & $9,3 \pm 0,03$ & $1,03 \pm 0,39$ & $7,43 \pm 0,06$ & $1,55 \pm 0,46$ \\
Bûches & $5,41 \pm 0,02$ & $1,11 \pm 0,10$ & $10,7 \pm 0,02$ & $0,84 \pm 0,27$ \\
Fourches & $12,22 \pm 0,04$ & $3,5 \pm 0,59$ & $9,95 \pm 0,05$ & $1,47 \pm 0,61$ \\
Petites charpentes & & & $14,6 \pm 0,01$ & $3,17 \pm 0,34$ \\
Grande charpente & $19,6 \pm 0,02$ & $3,55 \pm 0,48$ & $16,39 \pm 0,04$ & $3,15 \pm 0,62$ \\
Grume & $18,01 \pm 0,02$ & $2,25 \pm 0,61$ & $18,85 \pm 0,05$ & $1,73 \pm 0,14$ \\
Piquets & $10,54 \pm 0,02$ & $1,77 \pm 0,33$ & $7,61 \pm 0,04$ & $1,90 \pm 0,46$ \\
Ossatures & $4,23 \pm 0,01$ & $0,43 \pm 0,03$ & $2,81 \pm 0,01$ & $0,45 \pm 0,03$ \\
Traverses & $8,4 \pm 0,04$ & $1,92 \pm 0,51$ & $5,91 \pm 0,03$ & $2,04 \pm 0,55$ \\
\hline
\end{tabular}

Tableau 3 : Répartition des du type d'usages de bois dans les ménages au niveau des deux villages.

\begin{tabular}{lcc}
\hline Types d'usages & \multicolumn{2}{c}{ Villages } \\
\cline { 2 - 3 } & Arasofoa & Gaounawa \\
\hline Clôture & 69,52 & 67,24 \\
Construction des maisons & 18,88 & 28,38 \\
Bois de chauffe & 5,90 & 2,82 \\
Mobilier et ustensiles & 5,7 & 1,56 \\
Total & 100 & 100 \\
\hline
\end{tabular}

Tableau 4 : Volume $\left(\mathrm{m}^{3}\right)$ de bois pour chaque usage dans les deux villages.

\begin{tabular}{lcccc}
\hline Usages & \multicolumn{2}{c}{ Arasofoua } & \multicolumn{2}{c}{ Gaounawa } \\
\cline { 2 - 5 } & $\begin{array}{c}\text { Volume par } \\
\text { ménage }\left(\mathbf{m}^{\mathbf{3}}\right)\end{array}$ & $\begin{array}{c}\text { Volume total } \\
\left(\mathbf{m}^{\mathbf{3}}\right)\end{array}$ & $\begin{array}{c}\text { Volume par } \\
\text { ménage }\left(\mathbf{m}^{\mathbf{3}}\right)\end{array}$ & $\begin{array}{c}\text { Volume total } \\
\left(\mathbf{m}^{\mathbf{3}}\right)\end{array}$ \\
\hline Clôture & 296,06 & $2.897,55$ & 231,19 & $2.268,65$ \\
Construction des maisons & 38,42 & 375,11 & 40,95 & 364,47 \\
Bois de chauffe & 18,74 & 198,79 & 12,91 & 125,29 \\
Mobilier et ustensiles & 0,50 & 5,22 & 0,1973 & 1,873 \\
Total & 353,72 & 3476,67 & 285,25 & 2760,28 \\
\hline
\end{tabular}


Tableau 5 : Les espèces dont le bois est le plus utilisé au niveau des deux villages.

\begin{tabular}{lclc}
\hline \multicolumn{1}{c}{ Arasofoua } & \multicolumn{2}{c}{ Gaounawa } \\
\hline Espèces & Proportion (\%) & Espèces & Proportion (\%) \\
\hline Prosopis africana & 63,64 & Prosopis africana & 21,94 \\
Azadirachta indica & 13,55 & Azadirachta indica & 32,13 \\
Hyphaene thebaïca & 7,66 & Hyphaene thebaïca & 5,8 \\
Faidherbia albida & 2,14 & Faidherbia albida & 16,3 \\
Piliostigma reticulatum & 4,28 & Piliostigma reticulatum & 4,86 \\
Parkia biglobosa & 2,5 & Parkia biglobosa & 4,7 \\
Annona senegalensis & 2,14 & Annona senegalensis & 2,04 \\
Acacia nilotica & 0,89 & Acacia nilotica & 4,7 \\
Guiera senegalensis & 1,25 & Guiera senegalensis & 1,57 \\
Phoenix dactilifera & 1,25 & Phoenix dactilifera & 1,72 \\
Eucalyptus camaldulensis & 0,18 & Eucalyptus & 0,31 \\
& & camaldulensis & \\
Borassus aethiopum & 0,18 & Bauhinia rufescens & 0,47 \\
Psidium guajava & 0,18 & Psidium guajava & 0,47 \\
Sclerocarya birrea & 0,18 & Mangifera indica & 0,31 \\
Total & 100 & Tamarindus indica & 0,31 \\
& & Acacia senegal & 0,16 \\
& & Albizzia chevalieri & 0,16 \\
& & Ficus abutilifolia & 0,16 \\
& & Ficus sycomorus & 0,16 \\
& & Ficus sp & 0,16 \\
& & Total & 100 \\
\hline
\end{tabular}

Mode de transport du bois dans les villages

Trois modes de transport du bois existent dans les deux villages : essentiellement par charrettes, avec des ânes et sur la tête. Il ressort de l'enquête menée dans ces terroirs que l'approvisionnement en bois des ménages est de deux fagots d'environ $4 \mathrm{~kg}$ par jour s'il est transporté sur la tête et plus de cinq fagots par charrette. La fréquence de cet approvisionnement est au moins de deux fois par jour pour les piétons selon leur disponibilité et d'une fois pour les charretiers pendant la période de l'enquête (saison des pluies). Le transport se fait le plus souvent après les travaux champêtres durant les mois de janvier à mai. Le chef de famille ne tient pas compte du stock de bois déjà disponible autour de la maison. Le bois de chauffe est le plus utilisé.

\section{DISCUSSION}

Les interviewés ont souligné que les sécheresses des années 70 et 80 les ont motivés à protéger et à gérer les jeunes arbres de façon plus systématique et massive que par le passé. Les politiques nationales, inspirées de l'Engagement de Maradi (1984), ont favorisé la vulgarisation de la Régénération Naturelle Assistée. Le Projet de Renforcement des Services d'Appui à l'Agriculture (PRSAA 1988 - 1998) a formé des producteurs en matière de défrichement 
amélioré et de protection et entretien de la RNA. Pour réduire la perte du couvert arboré au Niger, la promotion de la RNA a été ainsi favorisée. Les espèces appréciées pour leur bois et les plus exploitées ont fait l'objet d'une gestion organisée communautairement dans les terroirs (Moustapha, 2004 ; PAFN, $2005 \mathrm{a}$ et b). Par cette pratique, les villages densément peuplé disposent aujourd'hui d'une densité importante d'arbres dans les champs (Larwanou et al., 2006). Pour s'approvisionner en bois, il n'est plus question de parcourir de longues distances. La plus grande disponibilité de bois dans les champs de cultures résulte de l'adoption massive de la RNA (Larwanou et al., 2006). Les paysans de chaque terroir villageois la pratiquent non seulement pour subvenir aux besoins de leur foyer (BOUGI, 2004), mais aussi pour les services écologiques rendus.

La consommation de bois de service utilisé dans la construction des habitats et autres usages est l'un des facteurs majeurs de l'exploitation des ressources ligneuses (Mahamane, 2004; Danguimbo, 2007). L'importante quantité de bois collectée provient des principales espèces du terroir (Ibeida, 2005), dont l'exploitation se déroule selon la méthode sélective. Cependant, ce choix sélectif n'est pas sans conséquence sur la diversité des espèces ligneuses. C'est le cas de $P$. africana, en recul dans la zone à cause de son bois imputrescible, très apprécié par les paysans mais aussi par le charbon qu'il produit pour les forges. Cette préférence du bois de service entraîne des coupes répétitives de certaines essences forestières et conduit même à l'épuisement de ces dernières.

Sur la base des résultats obtenus, il a été constaté que les produits (bois de service, bois de feu) provenant de la RNA ont réduit les difficultés que rencontrent les populations locales dans la recherche $\mathrm{du}$ bois. L'augmentation de la densité des ligneux dans les champs à travers la Régénération Naturelle
Assistée a eu un effet positif sur la vie des populations de cette zone.

Les éleveurs transhumants occasionnent des dégâts importants sur certaines espèces ligneuses comme $F$. albida qui est préférentiellement utilisé pour l'alimentation de leur bétail (Adam et al., 2006). La disponibilité du bois issu de la RNA pour les différents usages a permis un changement de l'environnement des communautés (Marthe, 2006). La présence de nombreux arbres dans les champs procure des revenus pour les ménages les plus pauvres à travers la vente du surplus de bois.

\section{Conclusion}

La protection et la gestion de la Régénération Naturelle Assistée par les producteurs sur leurs champs de culture et dans les jardins de la région de Zinder est un phénomène réel, dont l'ampleur est impressionnante. La RNA dépasse de loin les plantations artificielles, confirmant ainsi le potentiel productif des arbres hors forêt (Bellefontaine et al., 2002).

La protection systématique des jeunes plants (semis, rejets, drageons, marcottes terrestres) a eu comme effet la « construction» des parcs agroforestiers, qui ont contribué à maintenir ou à améliorer la fertilité des sols. Suite à cette évolution favorable, nombre de villages ont un parc encore jeune et les effets sur la fertilité des sols ne se font pas encore nettement sentir. N'empêche que presque partout les systèmes de production sont devenus plus complexes grâce à une meilleure intégration des cultures, du bétail et des ligneux.

Si la pratique de la RNA a contribué à un meilleur approvisionnement en bois des ménages, il n'en demeure pas moins qu'il reste encore des questionnements sur la pérennité de cet acquis. L'augmentation de la population dans cette zone nécessite toujours plus de bois pour satisfaire leurs besoins et il est temps que des dispositions juridiques 
soient prises afin de permettre une gestion soutenue de ces ressources. Une disposition de loi qui permettrait l'appropriation réelle et effective des arbres hors forêt à celui qui le protège dans un domaine privé semble une solution incontournable pour assurer la pérennité de ces ressources ligneuses.

\section{REMERCIEMENTS}

Nous tenons à remercier l'Institut International pour l'Environnement et le Développement (IIED) pour avoir financé cette étude. Nos remerciements vont aussi aux vaillantes populations des deux villages.

\section{REFERENCES}

Abdourahmane MS. 2009. Développement durable et impacts des politiques publiques de gestion de la vallée du fleuve Sénégal: du régional au local. VertigO - la Revue Electronique en Sciences de l'Environnement, 9(3) : 21.

Adam T, Abdoulaye T, Larwanou M, Yamba B, Reij C, Tappan G. 2006. Plus de gens, plus d'arbres : la transformation des systèmes de production au Niger et les impacts des investissements dans la gestion des ressources naturelles. Rapport de Synthèse Etude Sahel Niger Niamey: Comité Permanent Inter-Etats de Lutte contre la Sécheresse dans le Sahel (CILSS) et Université de Niamey.258p.

Awaiss, A. 2000. Gestion des forêts et des arbres au niveau des terroirs dans la région de Maradi. Drylands Research Working Paper 31, Crewkerne, England. $45 \mathrm{p}$.

Banoin M, Aristid J, Philippe J. 1994. Le défrichement amélioré au Sahel. Une pratique agroforestière adoptée par les paysans. Bois et Forêts des Tropiques, 225(1) : 31-43.

Bationo BA, Karim S, Bellefontaine R, Saadou M, Guinko S, Ichaou A, Bouhari A. 2005. Le marcottage terrestre: technique économique pour la régénération de certains ligneux tropicaux. Sécheresse, 16(4) : 309-11.

Bellefontaine R, Petit S, Pain-Orcet M, Deleporte Ph, Bertault JG. 2002. Trees outside forests. Towards better awareness. FAO Conservation Guide $\mathrm{n}^{\circ}$ 35, Rome, 2002, 234 p.

Bellefontaine R. 2005. Pour de nombreux ligneux, la reproduction sexuée n'est pas la seule voie : analyse de 875 cas - Texte introductif, tableau et bibliographie. Sécheresse, 16 (4) : 315-317.

Bertrand A, Babin D, Nasi R. 1999. L'adaptation de l'aménagement forestier à des situations diverses. Bois et Forêts des Tropiques, 261 (3) : 39 - 48.

Bonkano BH. 2005. Etude de la régénération naturelle dans les champs cultivés : cas du village de Dan Gamdji. Mémoire de fin d'étude/Ingénieur des Techniques Agricoles, Université Abdou Moumouni de Niamey, 65p.

Bougi CS. 2004. Analyse de quelques initiatives et innovations paysannes et leurs effets sur la sécurité alimentaire des ménages: cas des terroirs villageois de Damama et de El Gueza (Arrondissement d'Aguié/Maradi). Mémoire de fin d'étude/ Ingénieur des Techniques Agricoles /Faculté d'Agronomie, Université Abdou Moumouni de Niamey, $71 \mathrm{p}$.

Carucci R. 2000. Les arbres hors forêt: un moyen essentiel dans la lutte contre la désertification du Sahel. Unasylva, 51 : 19.

DanGuimbo I. 2007. Etude des facteurs socioéconomiques influant la biodiversité des systèmes des parcs agroforestiers dans le sud-ouest nigérien: cas des terroirs villageois de Boumba, Kotaki, Sorikoira, Gongueye et Djabbou. Mémoire DEA, Université Abdou Moumouni de Niamey, $131 \mathrm{p}$. 
Eckholm EP. 1975. The Other Energy Crisis: Firewood. Worldwatch Paper 1. Worldwatch Institute, Washington DC.

Ibeida T. 2005. Contribution à l'élaboration de tarif de cubage des espèces forestières des parcs agroforestiers de l'antenne de Magaria. Mémoire de fin d'étude Ingénieur des Techniques Agricoles /Faculté d'Agronomie, Université Abdou Moumouni de Niamey, $42 \mathrm{p}$.

Jesse CR. 1995. Africa Regional Study: Review of Policies in the Traditional Energy Sector (RPTES) Forestry Sector Policy Report in Senegal. Center for Population and Development Studies Harvard University, 93 p.

Joet A, Jouve P, Banoin M. 1998. Le défrichement amélioré au Sahel. Une pratique agroforestière adoptée par les paysans. Bois et Forêts des Tropiques, 255(5) : 31-43.

Jouve P. 1996. Gestion des Terroirs et des Ressources Naturelles au Sahel. CNEARC : Montpellier (France) ; 145 p.

Jouve P. 1991. Sécheresse au Sahel et stratégies paysannes. Sécheresse, 2 : 6169.

Larwanou M. 1998. Rapport d'activités, Institut National de la Recherche Agronomique du Niger, $57 \mathrm{p}$.

Larwanou M, Abdoulaye M, Chris R. 2006. Etude de la Régénération Naturelle Assistée dans la Région de Zinder (Niger); une Première Exploitation d'un Phénomène Spectaculaire. International Resources Group: Washington DC ; 56 p.

Larwanou M, Saadou M, Hamadou S. 2006. Les arbres dans les systèmes agraires en zone sahélienne du Niger: mode de gestion, atouts et contraintes. Tropicultura, 24 (1) : 14-18.

Mahaman IH. 2004. Etude de faisabilité d'un marché autour du palmier doum (Hyphaene thebaica) et de ses sousproduits dans le terroir villageois de El Gueza dans le département d'Aguié
(Maradi). Mémoire de fin d'étude/ Ingénieur des Techniques Agricoles /Faculté d'Agronomie, Université Abdou Moumouni de Niamey, $77 \mathrm{p}$.

Marou ZA, Abasse AT, Bokar M, Niang A, Cheick TO. 2002. Analyse de l'adoption de la Régénération Naturelle Assistée dans la région de Maradi au Niger. $2^{\mathrm{e}}$ Atelier régional sur les aspects socioéconomiques de l'Agroforesterie au Sahel, Bamako 4-6 mars 2002, 7 p.

Marthe DD. 2006. Restauration de l'environnement et changement sociaux de genre. Rapport d'études. CRESA, Niamey, CILSS et l'Université libre d'Amsterdam -Etude Sahélienne- Niger, $30 \mathrm{p}$.

Moustapha A. 2004. Etude de faisabilité technique et organisationnelle d'un marché de bois issu de la régénération naturelle dans le terroir villageois de Dan Saga. Mémoire de fin d'étude/Ingénieur des Techniques Agricoles/Faculté d'Agronomie, Université Abdou Moumouni de Niamey, 76 p.

PAFN. 2005a. Plan villageois d'aménagement des parcs agroforestiers de Ara Sofoua. Commune de Bandé. Département de Magaria. Ministère de l'Hydraulique de l'Environnement et de la Lutte contre la Désertification, Niamey, République du Niger, 29 p.

PAFN. 2005b. Plan villageois d'aménagement des parcs agroforestiers de Gaounawa. Commune de Bandé. Département de Magaria. Ministère de l'Hydraulique de l'Environnement et de la Lutte contre la Désertification, Niamey, République du Niger, 29 p.

Peltier R. 1994. Du reboisement imposé à la prise en compte des savoirs traditionnels. Nature Sciences Sociétés, 1(2): 67- 79.

Ministère des Finances et de l'Economie., 2001. Combien sommes-nous au Niger? Ministère des finances et de l'économie, secrétariat général, recensement général 
de la population et de l'habitat; juin 2003. République du Niger.

Saadou M. 1990. Propositions de subdivision phytogéographiques du Niger-Séminaire sur la recherche et le développement des ressources agro-sylvo-pastorales au sahel, RCS, Niamey, 1994, 48p.

Salissou A. 2004. Valorisation des produits et sous-produits ligneux dans la partie Nord d'Aguié : cas du terroir villageois de DanSaga. Mémoire de fin d'étude/ Ingénieur des Techniques Agricoles /Faculté d'Agronomie, Université Abdou Moumouni de Niamey, 84 p.
Winterbottom RT. 1980. Reforestation in the Sahel: problems and strategies. An analysis of the problem of deforestation and a review of the results of forestry projects in Upper Volta. The African Studies Association Annual Meeting, Philadelphia, October 15-18, 1980, 32 p.

Yamba B. 1994. Les ressources ligneuses et problèmes d'aménagement forestier dans la zone agricole du Niger. Thèse de Doctorat, Université Michel de Montaigne, Bordeaux III, 392p. 